\title{
Adaptive Q-S Synchronization of Fractional-Order Chaotic Systems with Nonidentical Structures
}

\author{
Li-xin Yang and Wan-sheng He \\ School of Mathematic and Statistics, Tianshui Normal University, Tianshui 741001, China \\ Correspondence should be addressed to Li-xin Yang; jiaodayanglixin@163.com
}

Received 27 July 2013; Accepted 17 August 2013

Academic Editor: Massimiliano Ferrara

Copyright ( 2013 L.-x. Yang and W.-s. He. This is an open access article distributed under the Creative Commons Attribution License, which permits unrestricted use, distribution, and reproduction in any medium, provided the original work is properly cited.

This paper investigates the adaptive $Q-S$ synchronization of the fractional-order chaotic systems with nonidentical structures. Based on the stability of fractional-order systems and adaptive control technique, a general formula for designing the controller and parameters update law is proposed to achieve adaptive Q-S synchronization between two different chaotic systems with different structures. The effective scheme parameters identification and Q-S synchronization of chaotic systems can be realized simultaneously. Furthermore, two typical illustrative numerical simulations are given to demonstrate the effectiveness of the proposed scheme, for each case, we design the controller and parameter update laws in detail. The numerical simulations are performed to verify the effectiveness of the theoretical results.

\section{Introduction}

Since the pioneering work of Pecora and Carroll, chaos control and synchronization have received particularly attention among scientists from various research fields including secure communication, and information science [1-4]. From then, many kinds of synchronization have been proposed in dynamical systems. For instance, Li et al. [5] pointed out that coexistence of complete synchronization in coupled identical Chen systems is by linear control. In [6], they presented an idea of stochastic phase synchronization about the dynamical evolution of the underlying system. Lu [7] proposed a new general scheme which was discussed for the generalized synchronization of discrete-time chaotic and hyperchaotic systems. Later on, Li et al. [8] studied antisynchronization of two different chaotic systems. In 1999, Mainieri and Rehacek first proposed the concept of projective synchronization which characterized that the drive and response systems could be synchronized up to a scaling constant matrix [9]. Recently, Li [10] proposed the Q-S synchronization that is a more general definition of projective synchronization when the response system contains scaling matrix. In the application of secure communication, more scaling matrices may also be a useful utility to improve the security of the secure communication scheme [11-16]. Amongst all kinds of chaos synchronization, projective synchronization is the most noticeable one because of its proportional feature between the synchronized dynamical states and, hence, it has received extensive research. To our best of knowledge, Q-S synchronization of the fractional-order chaotic systems has not considered adequately.

On the other hand, most of existing synchronization methods are mainly concerned with the synchronization of two special identical or similar or with mismatched systems. Zhang et al. [17] discussed the complete synchronization of a coupled fractional-order system, they have found a kind of interesting nonlinear phenomenon-hybrid synchronization in linearly coupled fractional-order chaotic systems. Recently, Mainieri and Rehacek [18] studied the hybrid projective synchronization of fractional-order chaotic system. They realized the slave system can be synchronized with the projection of the master system generated through state transformation. Despite these many results for the fractionalorder systems, but in fact, in many practical problems, the synchronization is carried out even though the oscillators have different structures; therefore, it is extremely necessary and important to study the synchronization of the fractionalorder systems with nonidentical structures. At the same time, 
most of the methods mentioned above have ignored the uncertainties, but in real situations, this assumption cannot be satisfied in many real situations because it is hard to known all the system parameters in advance [19-23]. In this case, the applications of the existing methods are somewhat limited. Hence, the synchronization and identification of chaotic systems with uncertainties are more essential work for research.

Motivated by the above discussion, this paper investigates the adaptive $Q-S$ synchronization of fractional-order chaotic systems via increased order. Moreover, based on the stability theory of fractional order systems, an adaptive synchronization controller and adaptive laws of parameters are developed; numerical simulations are carried out to demonstrate the effectiveness and flexibility for the controllers.

The organization of this paper is as follows. Preliminaries and model description are given in Section 2. In Section 3 based on the stability theory, a general Q-S synchronization approach of fractional-order chaotic systems with unknown parameters is presented. Section 4 shows the effectiveness of the approach for the extensive simulation studies. Finally, Section 5 concludes the paper.

\section{Preliminaries and Model Description}

The fractional calculus is a generalization of an integration and differentiation to a noninteger-order integro-differential operator which can be denoted by a fundamental operator as follows [23]:

$$
{ }_{a} D_{t}^{\alpha}= \begin{cases}\frac{d^{\alpha}}{d t^{\alpha}}, & R(\alpha)>0 \\ 1, & R(\alpha)=0 \\ \int_{\alpha}^{t}(d \tau)^{-\alpha}, & R(\alpha)<0 .\end{cases}
$$

There exist many definitions for fractional derivatives. The Riemann-Liouvile definition and the Caputo definition are the two most commonly used ones. In this paper, the Caputo definition is adopted for derivatives which is introduced as follows:

$$
D_{*}^{\alpha} f(t)=\frac{1}{\Gamma(n-\alpha)} \int_{t_{0}}^{t}(t-\tau)^{\alpha-n+1} f^{(n)}(\tau) d \tau,
$$

for $n-1 \leq \alpha<n$, where $\Gamma(\cdot)$ is the Gamma function,

$$
\Gamma(s)=\int_{0}^{\infty} t^{s-1} e^{-t} d t .
$$

The drive system and response system are described by

$$
\begin{gathered}
D_{*}^{q} \mathbf{x}=f(\mathbf{x}), \\
D_{*}^{q} \mathbf{y}=g(\mathbf{y})+U(t, \mathbf{x}, \mathbf{y}),
\end{gathered}
$$

where $\mathbf{x}, \mathbf{y}$ are the state vectors; $\mathbf{f}, \mathbf{g}$ are differentiable vector functions; and $\mathbf{U}(t, \mathbf{x}, \mathbf{y})$ is a controll function. We denote the vector error state be $\mathbf{e}=\mathbf{Q x}-\mathbf{S y}$, where $\mathbf{Q}, \mathbf{S}$ are scaling matrices.
Definition 1. For the given drive system (4) and response system (5), there exist two real matrices $\mathbf{Q}, \mathbf{S}$, such that $\lim _{t \rightarrow \infty}\|\mathbf{e}\|=\|\mathbf{Q} \mathbf{x}-\mathbf{S y}\|=0$. It is to say that the Q-S synchronization is achieved between system (4) and system (5).

Some remarks on Definition 1.

Remark 2. It is easy to see that the definition of Q-S synchronization encompasses complete synchronization, antisynchronization, and projective synchronization when matrices $Q$ and $S$ are selected special values, respectively. In short, Q-S synchronization is a more general form that includes many kinds of synchronization as its special items.

Remark 3. For simplicity for further discussion, we suppose that matrix $S$ is row full rank, which guarantee the inverse matrix exists when the matrix is a square matrix.

Lemma 4 (see [24]). For the nonlinear fractional-order system $D_{*}^{q} \mathbf{x}=f(\mathbf{x})$ or $D_{*}^{q} \mathbf{x}=\mathbf{A x}$ with the order as $0<q \leq 1$, if there exists a real symmetric positive definite matrix $\mathbf{P}$ such that the equation $J=\mathbf{x}^{T} \mathbf{P} D_{*}^{q} \mathbf{x} \leq 0$ always holds for any states $\mathbf{x}=$ $\left(x_{1}, x_{2}, \ldots, x_{n}\right) \in \mathbf{R}^{n}$, then the above fractional-order system is asymptotically locally stable.

\section{Theoretical Results}

Consider two general fractional-order uncertain chaotic systems which are referred to as the drive and response systems, respectively, in the form of

$$
\begin{gathered}
D_{*}^{q} \mathbf{x}=\mathbf{f}(\mathbf{x})+\mathbf{F}(\mathbf{x}) \boldsymbol{\Phi}, \\
D_{*}^{q} \mathbf{y}=\mathbf{g}(\mathbf{y})+\mathbf{G}(\mathbf{y}) \boldsymbol{\Theta}+\mathbf{U},
\end{gathered}
$$

where $\mathbf{x}=\left(x_{1}, x_{2}, \ldots, x_{n}\right)^{T} \in \mathbf{R}^{n_{1}}$ and $\mathbf{y}(t)=\left(y_{1}, y_{2}, \ldots\right.$, $\left.y_{n}\right)^{T} \in \mathbf{R}^{n_{2}}$ are the state vectors, $\mathbf{f}: \mathbf{R}^{n_{1}} \rightarrow \mathbf{R}^{n_{1}}$ and $\mathbf{g}: \mathbf{R}^{n_{2}} \rightarrow \mathbf{R}^{n_{2}}$ are continuous vector functions, including nonlinear terms, $\mathbf{F}: \mathbf{R}^{n_{1}} \rightarrow \mathbf{R}^{n_{1} \times m_{1}}$ and $\mathbf{G}: \mathbf{R}^{n_{2}} \rightarrow$ $\mathbf{R}^{n_{2} \times m_{2}}$ are matrix functions, $\boldsymbol{\Phi} \in \mathbf{R}^{m_{1}}$ and $\boldsymbol{\Theta} \in \mathbf{R}^{m_{2}}$ are the parameter vectors, and $\mathbf{U} \in \mathbf{R}^{n}$ is a controller to be designed later.

When $n_{1}=n_{2}, m_{1}=m_{2}$, the structure of the drive system is identical to that of the response system, this problem has been solved. Therefore, when the dimension of drive system is not equal to that of the response system, there is no doubt that it is an interesting problem. So we will investigate the following two cases.

Case $1\left(n_{1}>n_{2}\right)$. In this case, the dimension of the drive system is greater than that of the response system. For realizing the $Q-S$ synchronization between the drive system (6) and response system (7) with different structures, so we must add extra auxiliary state(s) to the response system.

We define the auxiliary state(s) $\mathbf{y}^{\prime} \in \mathbf{R}^{n_{1}-n_{2}}$ then we obtain a new state vector $\overline{\mathbf{y}}=\left(\mathbf{y}, \mathbf{y}^{\prime}\right)^{T}$, thus, the response system (7) is rewritten as follows:

$$
D_{*}^{q} \overline{\mathbf{y}}=\overline{\mathbf{g}}(\overline{\mathbf{y}})+\overline{\mathbf{G}}(\overline{\mathbf{y}}) \boldsymbol{\Theta}+\overline{\mathbf{U}}(t, x, y),
$$


where $\overline{\mathbf{y}}=\left(\begin{array}{c}\mathbf{y} \\ \mathbf{y}^{\prime}\end{array}\right), \overline{\mathbf{g}}(\overline{\mathbf{y}})=\left(\begin{array}{c}\mathbf{g}(\mathbf{y}) \\ 0\end{array}\right), \overline{\mathbf{G}}(\overline{\mathbf{y}})=\left(\begin{array}{c}\mathbf{G}(\mathbf{y}) \\ 0\end{array}\right), \overline{\mathbf{U}}(t, x, y)=$ $\left(\begin{array}{c}\mathbf{U}(t, x, y) \\ \mathbf{U}^{\prime}(t, x, y)\end{array}\right), \mathbf{y}^{\prime}$, and $\mathbf{U}^{\prime}(t, x, y) \in \mathbf{R}^{n_{1}-n_{2}}$.

Our goal is to design a suitable controller to realize the Q-S synchronization between drive system (6) and response system (8) with different arbitrary scaling matrices $Q, S$. The following theorem can be obtained.

Theorem 5. The Q-S synchronization of fractional-order systems between systems (6) and (8) can be achieved, if the active control under the hypothesis is given by (9) and the updating laws of the estimated parameter are given by (10)

$$
\begin{aligned}
\mathbf{U}=-\overline{\mathbf{g}}(\overline{\mathbf{y}})-\overline{\mathbf{G}}(\overline{\mathbf{y}}) \widehat{\boldsymbol{\Theta}}+\mathbf{S}^{-1} \mathbf{Q}(\mathbf{f}(\mathbf{x})+\mathbf{F}(\mathbf{x}) \widehat{\boldsymbol{\Phi}}+\mathbf{K e}), \\
D_{*}^{q} \widehat{\boldsymbol{\Phi}}=-[\mathbf{Q} \mathbf{F}(\mathbf{x})]^{T} \mathbf{e}, \\
D_{*}^{q} \widehat{\boldsymbol{\Theta}}=[\mathbf{S} \overline{\mathbf{G}}(\overline{\mathbf{y}})]^{T} \mathbf{e},
\end{aligned}
$$

where $\mathbf{K}=\operatorname{diag}\left(k_{1}, k_{2}, \ldots, k_{n}\right)$ is a gain matrix for each state controller, $\widehat{\boldsymbol{\Phi}}$ and $\widehat{\boldsymbol{\Theta}}$ are the estimated vectors of unknown parameters, and $\widetilde{\boldsymbol{\Phi}}=\boldsymbol{\Phi}-\widehat{\boldsymbol{\Phi}}, \widetilde{\boldsymbol{\Theta}}=\boldsymbol{\Theta}-\widehat{\boldsymbol{\Theta}}$.

Proof. We have the vector error state $\mathbf{e}=\mathbf{Q x}-\mathbf{S y}$, then we can obtain the error dynamical system as follows:

$$
D_{*}^{q} \mathbf{e}(t)=\mathbf{Q}(\mathbf{f}(\mathbf{x})+\mathbf{F}(\mathbf{x}) \boldsymbol{\Phi})-\mathbf{S}(\overline{\mathbf{g}}(\overline{\mathbf{y}})+\overline{\mathbf{G}}(\overline{\mathbf{y}}) \boldsymbol{\Theta}+\mathbf{U}) .
$$

According to Lemma 4, combining (9) and (10) with (11), one has

$$
\mathbf{J}=\left[\begin{array}{lll}
\mathbf{e}^{T} & \widetilde{\boldsymbol{\Phi}}^{T} & \widetilde{\boldsymbol{\Theta}}^{T}
\end{array}\right]\left[\begin{array}{c}
D_{*}^{q} \mathbf{e} \\
D_{*}^{q} \widetilde{\boldsymbol{\Phi}} \\
D_{*}^{q} \widetilde{\boldsymbol{\Theta}}
\end{array}\right]=\mathbf{e}^{T} D_{*}^{q} \mathbf{e}+\widetilde{\boldsymbol{\Phi}}^{T} D_{*}^{q} \widetilde{\boldsymbol{\Phi}}+\widetilde{\boldsymbol{\Theta}}^{T} D_{*}^{q} \widetilde{\boldsymbol{\Theta}} .
$$

From (6)-(11), we can get that

$$
\begin{aligned}
& \mathbf{e}^{T} D_{*}^{q} \mathbf{e}+\widetilde{\boldsymbol{\Phi}}^{T} D_{*}^{q} \widetilde{\boldsymbol{\Phi}}+\widetilde{\boldsymbol{\Theta}}^{T} D_{*}^{q} \widetilde{\boldsymbol{\Theta}} \\
& =\mathbf{e}^{T}[\mathbf{Q}(\mathbf{f}(\mathbf{x})+\mathbf{F}(\mathbf{x}) \boldsymbol{\Phi})-\mathbf{S}(\overline{\mathbf{g}}(\overline{\mathbf{y}})+\overline{\mathbf{G}}(\overline{\mathbf{y}}) \boldsymbol{\Theta}+\mathbf{U})] \\
& +\widetilde{\boldsymbol{\Phi}}^{T}\left([-\mathbf{Q F}(\mathbf{x})]^{T} \mathbf{e}\right)+\widetilde{\boldsymbol{\Theta}}^{T}\left([\mathbf{S} \overline{\mathbf{G}}(\overline{\mathbf{y}})]^{T} \mathbf{e}\right) \\
& =\mathbf{e}^{T}[\mathbf{Q}(\mathbf{f}(\mathbf{x})+\mathbf{F}(\mathbf{x}) \mathbf{\Phi})-\mathbf{S} \overline{\mathbf{g}}(\overline{\mathbf{y}})-\mathbf{S} \overline{\mathbf{G}}(\overline{\mathbf{y}}) \boldsymbol{\Theta}+\mathbf{S} \overline{\mathbf{g}}(\overline{\mathbf{y}}) \\
& \quad+\mathbf{S} \overline{\mathbf{G}}(\overline{\mathbf{y}})(\boldsymbol{\Theta}-\widetilde{\boldsymbol{\Theta}})-\mathbf{Q} \mathbf{f}(\mathbf{x})-\mathbf{Q F}(\mathbf{x})(\mathbf{\Phi}-\widetilde{\boldsymbol{\Phi}})-\mathbf{K e}] \\
& +\widetilde{\boldsymbol{\Phi}}^{T}\left(-(\mathbf{Q F}(\mathbf{x}))^{T} \mathbf{e}\right)+\widetilde{\boldsymbol{\Theta}}^{T}\left((\mathbf{S} \overline{\mathbf{G}}(\overline{\mathbf{y}}))^{T} \mathbf{e}\right) \\
& =-\mathbf{e}^{T} \mathbf{K e} .
\end{aligned}
$$

Suppose we select an appropriate matrix $\mathbf{K}$, such that $-\mathbf{e}^{T} \mathbf{K e} \leq 0$. From Lemma 4 , the origin of error dynamical system (11) is asymptotically stable, it is to say that the response system (6) can synchronize the drive system (8) globally and asymptotically.
Remark 6. In order to identify the unknown parameters, we suppose that the nonlinear vector functions $-[\mathbf{Q F}(\mathbf{x})]^{T}$ and $[\mathbf{S G}(\overline{\mathbf{y}})]^{T}$ should be linearly independent.

Based on Theorem 5, two corollaries can be easily derived as below.

Corollary 7. Suppose the parameters in the drive system $\Phi$ are known, the controller can be designed as follows:

$$
\mathbf{U}=-\overline{\mathbf{g}}(\overline{\mathbf{y}})-\overline{\mathbf{G}}(\overline{\mathbf{y}}) \widehat{\boldsymbol{\Theta}}+\mathbf{S}^{-1} \mathbf{Q}(\mathbf{f}(\mathbf{x})+\mathbf{F}(\mathbf{x}) \boldsymbol{\Phi}+\mathbf{K e}) .
$$

Moreover, the parameter update laws are degraded as

$$
D_{*}^{q} \widehat{\boldsymbol{\Theta}}=[\mathbf{S} \overline{\mathbf{G}}(\overline{\mathbf{y}})]^{T} \mathbf{e} .
$$

Therefore, the drive system and response system can achieve Q$S$ synchronization.

Corollary 8. Suppose the parameters $\Theta$ in the response system are known, then the controller is modified as

$$
\mathbf{U}=-\overline{\mathbf{g}}(\overline{\mathbf{y}})-\overline{\mathbf{G}}(\overline{\mathbf{y}}) \boldsymbol{\Theta}+\mathbf{S}^{-1} \mathbf{Q}(\mathbf{f}(\mathbf{x})+\mathbf{F}(\mathbf{x}) \widehat{\Phi}+\mathbf{K e}) .
$$

Moreover, the parameter update laws can be taken as

$$
D_{*}^{q} \widehat{\boldsymbol{\Phi}}=-[\mathbf{Q F}(\mathbf{x})]^{T} \mathbf{e} .
$$

Therefore, the drive system and response system can achieve Q$S$ synchronization.

Case $2\left(n_{1}<n_{2}\right)$. That is, since the order of the drive system is lower than that of the response system, the redundant state(s) in the response system should synchronize other artificially built state(s) of the drive system.

Denote the auxiliary state as $\mathbf{x}^{\prime} \in \mathbf{R}^{n_{2}-n_{1}}$, then we can get a new $n_{2}$ dimension state vector $\overline{\mathbf{x}}=\left(\begin{array}{c}\mathbf{x} \\ \mathbf{x}^{\prime}\end{array}\right)$, and the new drive system can rewrite as

$$
D_{*}^{q} \overline{\mathbf{x}}=\overline{\mathbf{f}}_{1}(\overline{\mathbf{x}})+\overline{\mathbf{F}}_{1}(\overline{\mathbf{x}}) \boldsymbol{\Theta},
$$

where $\overline{\mathbf{x}}=\left(\begin{array}{c}\mathbf{x} \\ \mathbf{x}^{\prime}\end{array}\right), \overline{\mathbf{f}}_{1}(\mathbf{x})=\left(\begin{array}{c}\mathbf{f}_{1}(\mathbf{x}) \\ \mathbf{f}_{1}^{\prime}(\mathbf{x})\end{array}\right)$, and $\overline{\mathbf{F}}_{1}(\mathbf{x})=\left(\begin{array}{c}\mathbf{F}_{1}(\mathbf{x}) \\ \mathbf{F}_{1}^{\prime}(\mathbf{x})\end{array}\right)$.

Theorem 9. For the given scaling matrices $Q, S$, the Q-S synchronization of fractional-order systems between system (6) and (8) can be achieved, if the active control under the hypothesis is given by (19) and the updating laws of the estimated parameter are given by (20)

$$
\begin{aligned}
\mathbf{U}=-g(\mathbf{y})-\mathbf{G}(\mathbf{y}) \widehat{\boldsymbol{\Theta}}+\mathbf{S}^{-1}(\mathbf{Q}(\overline{\mathbf{f}}(\overline{\mathbf{x}})+\overline{\mathbf{F}}(\overline{\mathbf{x}}) \widehat{\boldsymbol{\Phi}})+\mathbf{K e}), \\
D_{*}^{q} \widehat{\boldsymbol{\Phi}}=-[\mathbf{Q} \overline{\mathbf{F}}(\mathbf{x})]^{T} \mathbf{e} \\
D_{*}^{q} \widehat{\boldsymbol{\Theta}}=[\mathbf{S G}(\mathbf{y})]^{T} \mathbf{e} .
\end{aligned}
$$

The proof is the same as in Case 1 , so we omit it.

Similarly, from Theorem 9, we can also obtain the following corollaries. 
Corollary 10. Suppose the parameters in the drive system $\Phi$ are known, the controller can be designed as follows:

$$
\mathbf{U}=-g(\mathbf{y})-\mathbf{G}(\mathbf{y}) \widehat{\boldsymbol{\Theta}}+\mathbf{S}^{-1}(\mathbf{Q}(\overline{\mathbf{f}}(\overline{\mathbf{x}})+\overline{\mathbf{F}}(\overline{\mathbf{x}}) \mathbf{\Phi})+\mathbf{K e}) .
$$

Moreover, the parameter update laws are degraded as

$$
D_{*}^{q} \widehat{\boldsymbol{\Theta}}=[\mathbf{S G}(\mathbf{y})]^{T} \mathbf{e} .
$$

Therefore, the drive system and response system can achieve Q$S$ synchronization.

Corollary 11. Suppose the parameters $\Theta$ in the response system are known, then the controller is modified as

$$
\mathbf{U}=-g(\mathbf{y})-\mathbf{G}(\mathbf{y}) \boldsymbol{\Theta}+\mathbf{S}^{-1}(\mathbf{Q}(\overline{\mathbf{f}}(\overline{\mathbf{x}})+\overline{\mathbf{F}}(\overline{\mathbf{x}}) \widehat{\boldsymbol{\Phi}})+\mathbf{K e}) .
$$

Moreover, the parameter update laws can be taken as

$$
D_{*}^{q} \widehat{\mathbf{\Phi}}=-[\mathbf{Q} \overline{\mathbf{F}}(\mathbf{x})]^{T} \mathbf{e} .
$$

Therefore, the drive system and response system can achieve the adaptive Q-S synchronization.

\section{Simulation Results}

In this section, we will present two numerical examples to verify the effectiveness of the adaptive Q-S synchronization scheme with different structures proposed in Section 3. All the following numerical simulations are performed via the predictor-corrector algorithm [23].

4.1. Adaptive Q-S Synchronization between the FractionalOrder Hyperchaotic Lorenz System and Fractional-Order Financial System $\left(n_{1}>n_{2}\right)$. The fractional-order Lorenz system can be described as follows:

$$
\begin{gathered}
D_{*}^{q} x_{1}=a\left(x_{2}-x_{1}\right)+x_{4}, \\
D_{*}^{q} x_{2}=c x_{1}-x_{2}-x_{1} x_{3}, \\
D_{*}^{q} x_{3}=x_{1} x_{2}-b x_{3}, \\
D_{*}^{q} x_{4}=-x_{2} x_{3}+r x_{4},
\end{gathered}
$$

when $q=0.98$ and $(a, b, c, r)=(10,8 / 3,28,-1)$, the fractional-order Lorenz displays hyperchaotic attractors.

The fractional-order financial system is described by

$$
\begin{gathered}
D_{*}^{q} y_{1}=y_{3}+\left(y_{2}-d\right) y_{1}, \\
D_{*}^{q} y_{2}=1-f y_{2}-y_{1}^{2}, \\
D_{*}^{q} y_{3}=-y_{1}-h y_{3},
\end{gathered}
$$

when $q=0.98,(d, f, h)=(3,0.1,1)$, and the fractional-order financial system shows chaotic attractors.
We take the fractional-order Lorenz system as drive system, then the response system is given as

$$
\begin{gathered}
D_{*}^{q} y_{1}=y_{3}+\left(y_{2}-d\right) y_{1}+u_{1}, \\
D_{*}^{q} y_{2}=1-f y_{2}-y_{1}^{2}+u_{2}, \\
D_{*}^{q} y_{3}=-y_{1}+h y_{3}+u_{3} .
\end{gathered}
$$

In order to realize the $Q-S$ synchronization between the above two different structure systems, we construct an auxiliary state $y_{4}=0$ to the response system, so the response system can be rewritten as the following form:

$$
\begin{gathered}
D_{*}^{q} y_{1}=y_{3}+\left(y_{2}-d\right) y_{1}+u_{1}, \\
D_{*}^{q} y_{2}=1-f y_{2}-y_{1}^{2}+u_{2}, \\
D_{*}^{q} y_{3}=-y_{1}-h y_{3}+u_{3}, \\
D_{*}^{q} y_{4}=u_{4} .
\end{gathered}
$$

For simplicity, we choose the matrices $Q=S=\left[\begin{array}{llll}1 & 0 & 0 & 0 \\ 0 & 1 & 0 & 0 \\ 0 & 0 & 1 & 0 \\ 0 & 0 & 0 & 1\end{array}\right]$, and $S^{-1}=\left[\begin{array}{llll}1 & 0 & 0 & 0 \\ 0 & 1 & 0 & 0 \\ 0 & 0 & 1 & 0 \\ 0 & 0 & 0 & 1\end{array}\right]$.

From the prefer theorem, we can obtain that

$$
\mathbf{f}(\mathbf{x})=\left[\begin{array}{c}
x_{4} \\
x_{2}-x_{1} x_{3} \\
x_{1} x_{2} \\
-x_{2} x_{3}
\end{array}\right]
$$$$
\mathbf{F}(\mathbf{x})=\left[\begin{array}{cccc}
x_{2}-x_{1} & 0 & 0 & 0 \\
0 & 0 & x_{1} & 0 \\
0 & x_{3} & 0 & 0 \\
0 & 0 & 0 & x_{4}
\end{array}\right], \quad \boldsymbol{\Phi}=\left[\begin{array}{l}
a \\
b \\
c \\
r
\end{array}\right],
$$$$
g(\overline{\mathbf{y}})=\left[\begin{array}{c}
y_{3}+y_{2} y_{1} \\
1-y_{1}^{2} \\
-y_{1} \\
0
\end{array}\right]
$$

$$
G(\overline{\mathbf{y}})=\left[\begin{array}{cccc}
-y_{1} & 0 & 0 & 0 \\
0 & -y_{2} & 0 & 0 \\
0 & 0 & -y_{3} & 0 \\
0 & 0 & 0 & 0
\end{array}\right], \quad \boldsymbol{\Theta}=\left[\begin{array}{l}
d \\
f \\
h \\
0
\end{array}\right] .
$$

According to Definition 1, we choose the following scaling matrices:

$$
\mathbf{Q} \mathbf{x}=\left[\begin{array}{c}
x_{1} \\
x_{2} \\
x_{3} \\
1
\end{array}\right], \quad \mathbf{S y}=\left(\begin{array}{c}
y_{1} \\
2 y_{2}+y_{3} \\
2 y_{3} \\
y_{4}
\end{array}\right)
$$




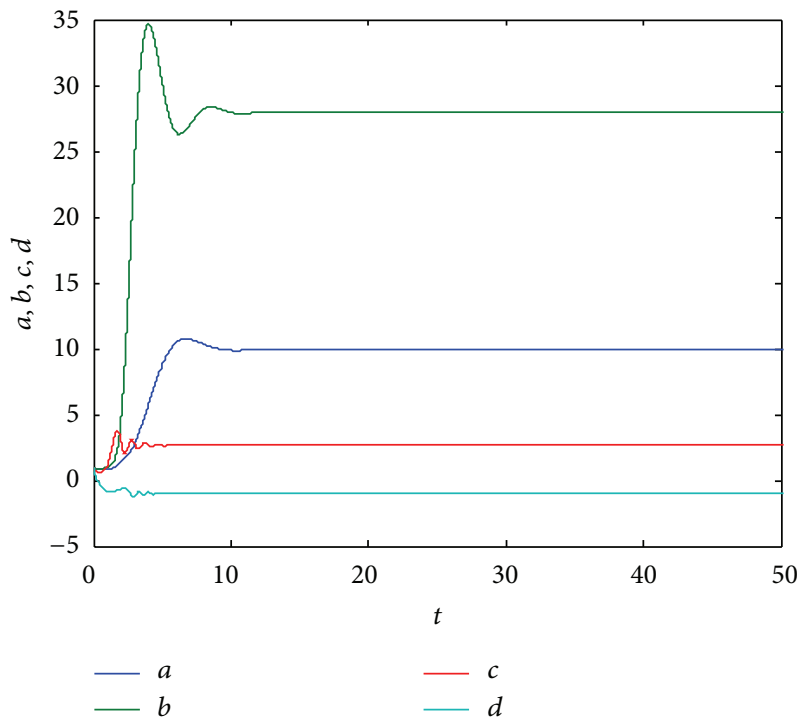

FIGURE 1: Time evolution of parameter estimations for system (25).

From (9), we have

$$
\begin{gathered}
u_{1}=-y_{3}-y_{1} y_{2}+\widehat{d} y_{1}+x_{4}+\widehat{a}\left(x_{2}-x_{4}\right)+k_{1} e_{1}, \\
u_{2}=y_{1}^{2}-1+\widehat{f} y_{2}-x_{2}-x_{1} x_{3}+\widehat{c} x_{1}+k_{2} e_{2}, \\
u_{3}=y_{1}+x_{1} x_{2}-\widehat{h} y_{3}-\widehat{b} x_{3}+k_{3} e_{3} \\
u_{4}=-x_{2} x_{3}+\widehat{r} x_{4}+k_{4} e_{4}
\end{gathered}
$$

and the parameters estimation update laws as follows:

$$
\begin{gathered}
D_{*}^{q} \widehat{a}=\left(x_{4}-x_{2}\right) e_{1}, \\
D_{*}^{q} \widehat{b}=x_{3} e_{3}, \\
D_{*}^{q} \widehat{c}=-x_{1} e_{2}, \\
D_{*}^{q} \widehat{r}=-x_{4} e_{4}, \\
D_{*}^{q} \widehat{d}=y_{1} e_{1}, \\
D_{*}^{q} \widehat{f}=y_{2} e_{2}, \\
D_{*}^{q} \widehat{h}=y_{3} e_{3} .
\end{gathered}
$$

It selects the parameters of the drive and response systems as $(a, b, c, r)=(10,8 / 3,28,-1), d=3, f=0.1$, and $h=$ 1 to ensure the chaotic behavior, the initial conditions are $\left(x_{1}(0), x_{2}(0), x_{3}(0)\right)=(1,2,3)$ and $\left(y_{1}(0), y_{2}(0), y_{3}(0)\right)=$ $(0.1,0.1,0.1)$, and the initial values for the estimations of unknown parameters are $\widehat{a}=1, \widehat{b}=1, \widehat{c}=1, \widehat{r}=$ $1, \widehat{d}=0.1, \widehat{f}=0.1, \widehat{h}=0.1$, respectively. The feedback gain $\mathbf{K}=(1,1,1,1)$. Figures 1 and 2 show that all the unknown parameters in the system are identified to their true values as time increase. It is shown in Figure 3 that the synchronization errors between the two different structures

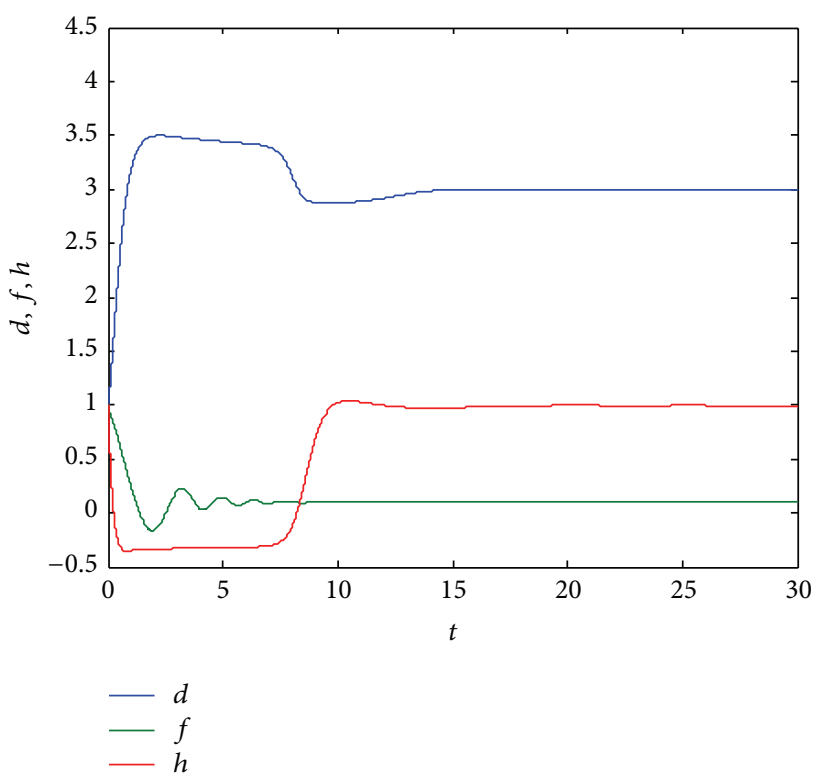

FIGURE 2: Time evolution of parameter estimations for system (27).

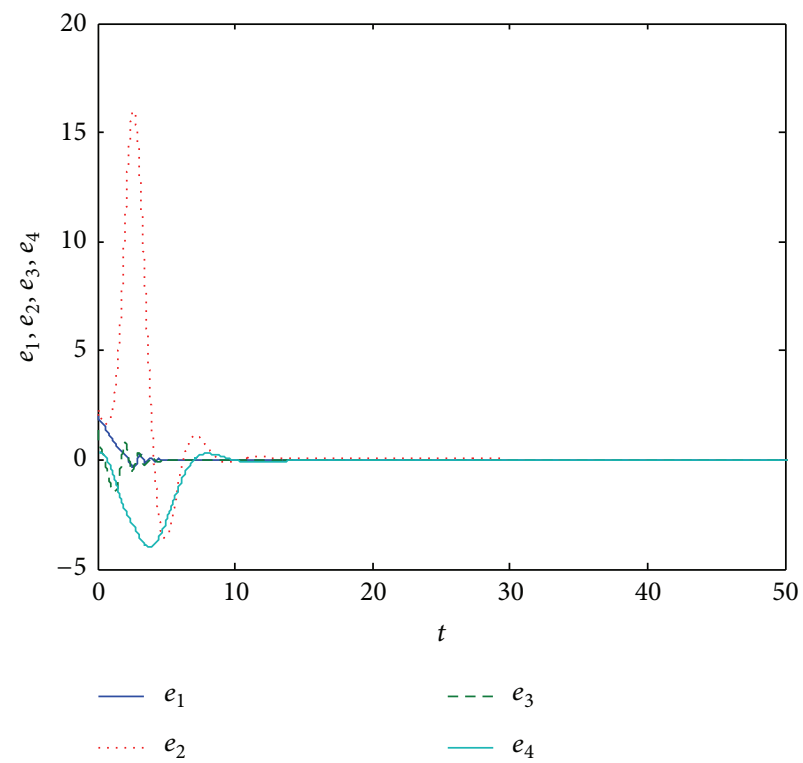

FIGURE 3: Time evolution of synchronization error between system (25) and system (27).

fractional-order systems (25) and (27) converge to zero with time passing.

4.2. Adaptive Q-S Synchronization between Arneodo System and Hyperchaotic Chen System $\left(n_{1}<n_{2}\right)$. In this subsection, we will study the Q-S synchronization between Arneodo system and hyperchaotic Chen system, the drive system is Arneodo system and the response system is hyperchaotic Chen system. 
The fractional order Arneodo system are described by

$$
\begin{gathered}
D_{*}^{q} x_{1}=x_{2}, \\
D_{*}^{q} x_{2}=x_{3}, \\
D_{*}^{q} x_{3}=-\beta_{1} x_{1}-\beta_{2} x_{2}-\beta_{3} x_{3}+\beta_{4} x_{1}^{3},
\end{gathered}
$$

where the true values for each parameter are $\left(\beta_{1}, \beta_{2}, \beta_{3}, \beta_{4}\right)=$ $(-5.5,3.5,1,-1)$ and the order $q=0.97$, system (33) displays chaotic behaviors.

The controlled hyperchaotic fractional-order Chen system is described as

$$
\begin{gathered}
D_{*}^{q} y_{1}=a_{1}\left(y_{2}-y_{1}\right)+y_{4}+u_{1}, \\
D_{*}^{q} y_{2}=b_{1} y_{1}-y_{1} y_{3}+c_{1} y_{2}+u_{2}, \\
D_{*}^{q} y_{3}=y_{1} y_{2}-d_{1} y_{3}+u_{3}, \\
D_{*}^{q} y_{4}=y_{2} y_{3}+0.5 y_{4}+u_{4} .
\end{gathered}
$$

Based on the proposed scheme in Section 3, we should add an auxiliary state variable to the drive system.

For the convenience of controller form, we can get

$$
\begin{gathered}
\mathbf{f}(\overline{\mathbf{x}})=\left[\begin{array}{c}
x_{2} \\
x_{3} \\
0 \\
1
\end{array}\right], \quad \mathbf{F}(\overline{\mathbf{x}})=\left[\begin{array}{cccc}
0 & 0 & 0 & 0 \\
0 & 0 & 0 & 0 \\
-x_{1} & -x_{2} & -x_{3} & x_{1}^{3} \\
0 & 0 & 0 & 0
\end{array}\right], \\
\mathbf{\Phi}=\left[\begin{array}{l}
\beta_{1} \\
\beta_{2} \\
\beta_{3} \\
\beta_{4}
\end{array}\right], \\
g(\mathbf{y})=\left[\begin{array}{c}
y_{4} \\
-y_{1} y_{3}-y_{2} \\
y_{1} y_{2} \\
y_{2} y_{3}+0.5 y_{4}
\end{array}\right], \\
G(\mathbf{y})=\left[\begin{array}{cccc}
y_{2}-y_{1} & 0 & 0 & 0 \\
0 & y_{1} & y_{2} & 0 \\
0 & 0 & 0 & -y_{3} \\
0 & 0 & 0 & 0
\end{array}\right], \quad \Theta=\left[\begin{array}{l}
a_{1} \\
b_{1} \\
c_{1} \\
d_{1}
\end{array}\right] .
\end{gathered}
$$

According to Definition 1, we choose the scaling matrices

$$
\mathbf{Q} \mathbf{x}=\left[\begin{array}{c}
x_{1} \\
x_{2} \\
x_{3} \\
1
\end{array}\right], \quad \mathbf{S y}=\left(\begin{array}{c}
y_{1} \\
2 y_{2}+y_{3} \\
2 y_{3} \\
y_{4}
\end{array}\right)
$$

So we can obtain

$$
\begin{gathered}
\mathbf{Q}=\left[\begin{array}{llll}
1 & 0 & 0 & 0 \\
0 & 1 & 0 & 0 \\
0 & 0 & 1 & 0 \\
0 & 0 & 0 & 1
\end{array}\right], \quad \mathbf{S}=\left[\begin{array}{cccc}
1 & 0 & 0 & 0 \\
0 & 1 & 1 & 0 \\
0 & 0 & 2 & 0 \\
0 & 0 & 0 & -1
\end{array}\right], \\
\mathbf{S}^{-1}=\left[\begin{array}{cccc}
-1 & 0 & 0 & 0 \\
-1 & -1 & \frac{1}{2} & 1 \\
0 & 0 & 0 & 0 \\
0 & 0 & 0 & 1
\end{array}\right] .
\end{gathered}
$$

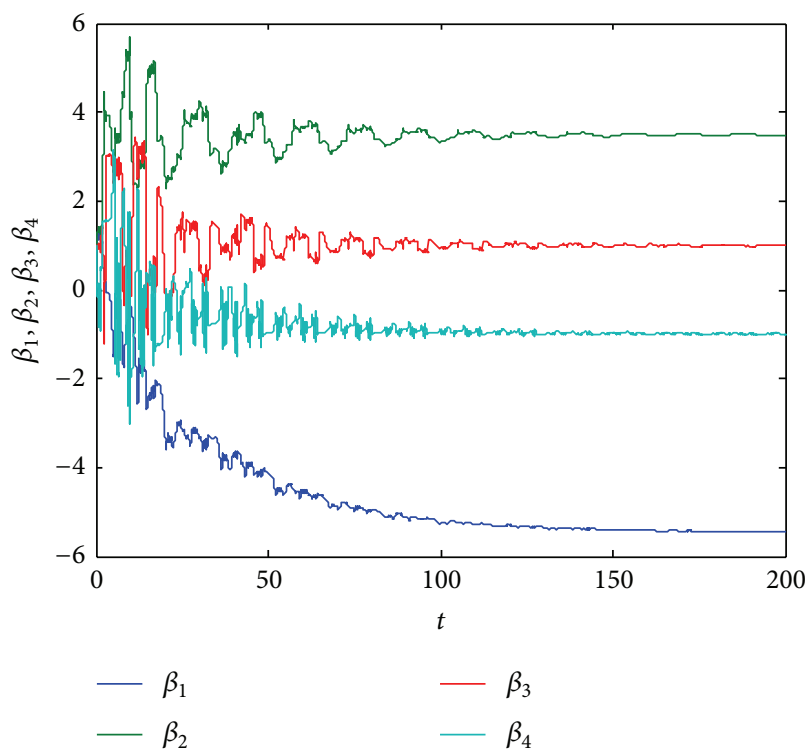

FIgURE 4: Time evolution of parameter estimations for system (33).

Based on the proposed scheme, the controllers are designed in the following form:

$$
\begin{gathered}
u_{1}=-y_{4}-\left(y_{2}-y_{1}\right) \widehat{a}_{1}-x_{2}+k_{1} e_{1}, \\
u_{2}=y_{1} y_{3}-\widehat{b}_{1} y_{1}-\widehat{c}_{1} y_{2}-x_{2}-x_{3}+1+k_{2} e_{2}, \\
u_{3}=-y_{1} y_{2}+y_{3} \widehat{d}_{1}-x_{1} \widehat{\beta}_{1}-x_{2} \widehat{\beta}_{2}-x_{3} \widehat{\beta}_{3}+x_{1}^{3} \widehat{\beta}_{4}+k_{3} e_{3}, \\
u_{4}=-y_{2} y_{3}-\widehat{r} y_{4}+1+k_{4} e_{4},
\end{gathered}
$$

and the parameters estimation update laws as follows:

$$
\begin{gathered}
D_{*}^{q} \widehat{a}_{1}=-\left(y_{2}-y_{1}\right) e_{1}, \\
D_{*}^{q} \widehat{b}_{1}=-y_{1} e_{2}, \\
D_{*}^{q} \widehat{c}_{1}=-y_{2} e_{2}, \\
D_{*}^{q} \widehat{d}_{1}=-2 y_{3} e_{3}, \\
D_{*}^{q} \widehat{\beta}_{1}=x_{1} e_{3}, \\
D_{*}^{q} \widehat{\beta}_{2}=x_{2} e_{3}, \\
D_{*}^{q} \widehat{\beta}_{3}=x_{3} e_{3}, \\
D_{*}^{q} \widehat{\beta}_{4}=-x_{1}^{3} e_{3} .
\end{gathered}
$$

Analogously, we also would like to give the numerical simulations to verify the effectiveness of the abovedesigned controller and the update laws. In these numerical simulations, we take the initial states as the initial conditions are $\left(x_{1}(0), x_{2}(0), x_{3}(0)\right)=(1,-2,5)$ and $\left(y_{1}(0), y_{2}(0)\right.$, $\left.y_{3}(0)\right)=(1,1,1)$, and the initial values for the estimations of unknown parameters are $\widehat{\beta}_{1}=1, \widehat{\beta}_{2}=1, \widehat{\beta}_{3}=1, \widehat{\beta}_{4}=$ $1, \widehat{a}_{1}=1, \widehat{b}_{1}=0.1, \widehat{c}_{1}=1, \widehat{d}_{1}=0.1$. The feedback gain $\mathbf{K}=$ $(5,5,5,5)$. From Figures 4 and 5 , it can be clearly seen that all 


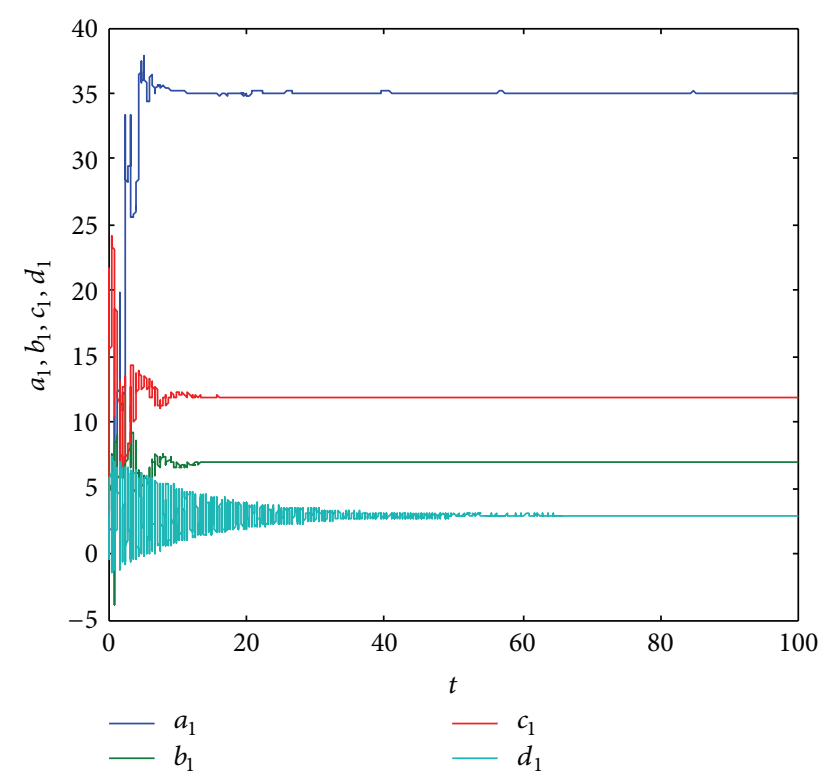

Figure 5: Time evolution of parameter estimations for system (34).

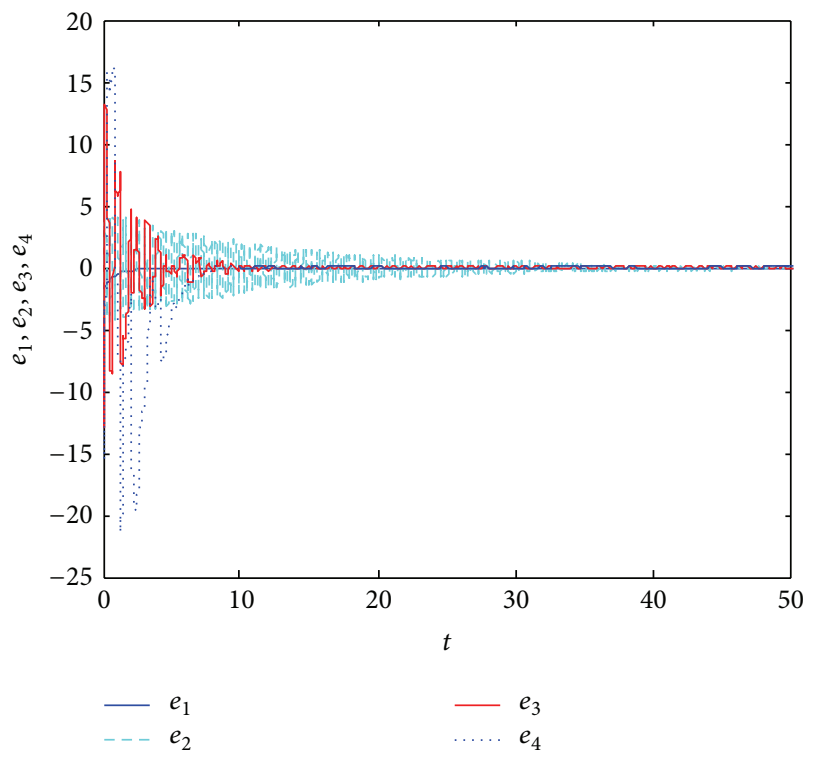

FIGURE 6: Time evolution of synchronization error between system (33) and system (34).

the unknown parameters in the drive and response systems are identified to their true values as time increase. It is shown in Figure 6 that the synchronization errors between the two different structures fractional-order systems (33) and (34) converge to zero with time passing, which means that the Q$S$ synchronization between the fractional-order system (33) and system (34) with different dimension is achieved.

\section{Conclusion}

In this paper, adaptive Q-S synchronization of fractionalorder chaotic systems with different structures is investigated. Based on the stability theory and adaptive control method, a general approach for suitable controller and adaptive laws is provided to realize the Q-S synchronization. Typical examples are taken to display the applications of the proposed scheme. For each case, the controller and parameter update laws are designed in detail. Meanwhile, results could extend to other fractional-chaotic systems with different structure and uncertain parameters. Numerical simulations show the effectiveness and feasibility of the controllers and identification rules.

\section{Acknowledgments}

The authors would like to thank the anonymous reviewers for their valuable comments and suggestions in helping them improve this paper.

\section{References}

[1] G. W. Leibniz, Mathematics Schiften, Georg Olms Verlagsbuchhandlung, Hilesheim, Germany, 1962.

[2] A. L. Chian, F. A. Borotto, E. L. Rempel, and C. Rogers, "Attractor merging crisis in chaotic business cycles," Chaos, Solitons and Fractals, vol. 24, no. 3, pp. 869-875, 2005.

[3] A. L. Chian, E. L. Rempel, and C. Rogers, "Complex economic dynamics: chaotic saddle, crisis and intermittency," Chaos, Solitons and Fractals, vol. 29, no. 5, pp. 1194-1218, 2006.

[4] K. Sasakura, "On the dynamic behavior of Schinasi's business cycle model," Journal of Macroeconomics, vol. 16, no. 3, pp. 423444, 1994.

[5] C. D. Li, Q. Chen, and T. Huang, "Coexistence of anti-phase and complete synchronization in coupled chen system via a single variable," Chaos, Solitons and Fractals, vol. 38, no. 2, pp. 461464, 2008.

[6] I. Osorio and Y. Lai, "A phase-synchronization and randommatrix based approach to multichannel time-series analysis with application to epilepsy," Chaos, vol. 21, no. 3, pp. 3310-3318, 2011.

[7] J. F. Lu, "Generalized (complete, lag, anticipated) synchronization of discrete-time chaotic systems," Communications in Nonlinear Science and Numerical Simulation, vol. 13, no. 9, pp. 1851-1859, 2008.

[8] W. L. Li, X. Q. Chen, and Z. P. Shen, "Anti-synchronization of two different chaotic systems," Physica A, vol. 387, no. 14, pp. 3747-3757, 2008

[9] R. Mainieri and J. Rehacek, "Projective synchronization in three-dimensional chaotic systems," Physical Review Letters, vol. 82, no. 15, pp. 3042-3045, 1999.

[10] G. H. Li, "Modified projective synchronization of chaotic system," Chaos, Solitons and Fractals, vol. 32, no. 5, pp. 17861790, 2007.

[11] M. Ferrara, F. Munteanu, C. Udriste, and D. Zugravescu, "Controllability of a nonholonomic macroeconomic system," Journal of Optimization Theory and Applications, no. 154, pp. 1036-1054, 2012

[12] B. M. Vinagre and V. Feliu, "Modeling and control of dynamic systems using fractional calculus: application to electrochemical processes and flexible structures," in Proceedings of the 41st IEEE Conference on Decision and Control, Las Vegas, Nev, USA, 2002. 
[13] A. D. Mengue and B. Z. Essimbi, "Secure communication using chaotic synchronization in mutually coupled semiconductor lasers," Nonlinear Dynamics, vol. 70, no. 4, pp. 1241-1253, 2012.

[14] M. Ferrara and L. Guerrini, "On the dynamics of a three-sector growth model," International Review of Economics, vol. 55, no. 3, pp. 275-284, 2008.

[15] J. Huang, "Chaos synchronization between two novel different hyperchaotic systems with unknown parameters," Nonlinear Analysis, Theory, Methods and Applications, vol. 69, no. 11, pp. 4174-4181, 2008.

[16] L. Liu, D. L. Liang, and C. X. Liu, "Nonlinear state-observer control for projective synchronization of a fractional-order hyperchaotic system," Nonlinear Dynamics, vol. 69, no. 3, pp. 1929-1939, 2012.

[17] J. F. Zhang, Q. Pei, and X. L. Zhang, "A new kind of nonlinear phenomenon in coupled fractional-order chaotic systems: coexistence of anti-phase and complete synchronization," Chinese Physics B, vol. 20, no. 8, Article ID 080503, pp. 162-168, 2011.

[18] R. Mainieri and J. Rehacek, "Projective synchronization in three-dimensional chaotic systems," Physical Review Letters, vol. 82, no. 15, pp. 3042-3045, 1999.

[19] S. Wang, Y. G. Yu, and M. Diao, "Hybrid projective synchronization of chaotic fractional order systems with different dimensions," Physica A, vol. 389, no. 21, pp. 4981-4988, 2010.

[20] W. L. He, F. Qian, J. D. Cao, and Q. L. Han, "Impulsive synchronization of two nonidentical chaotic systems with timevarying delay," Physics Letters A, vol. 375, no. 3, pp. 498-504, 2011.

[21] X. F. Li, A. C. Leung, X. J. Liu, X. P. Han, and Y. Chu, "Adaptive synchronization of identical chaotic and hyper-chaotic systems with uncertain parameters," Nonlinear Analysis: Real World Applications, vol. 11, no. 4, pp. 2215-2223, 2010.

[22] X. Y. Wang and J. M. Song, "Synchronization of the fractional order hyperchaos Lorenz systems with activation feedback control," Communications in Nonlinear Science and Numerical Simulation, vol. 14, no. 8, pp. 3351-3357, 2009.

[23] K. Diethelm, N. J. Ford, and A. D. Freed, "A predictor-corrector approach for the numerical solution of fractional differential equations," Nonlinear Dynamics, vol. 29, no. 1-4, pp. 3-22, 2002.

[24] J. B. Hu, Y. Han, and L. D. Zhao, "A novel stablility theorem for fractional systems and its applying in synchronizing fractional chaotic system based on back-stepping approach," Acta Physica Sinica, vol. 58, no. 4, pp. 2235-2239, 2009. 


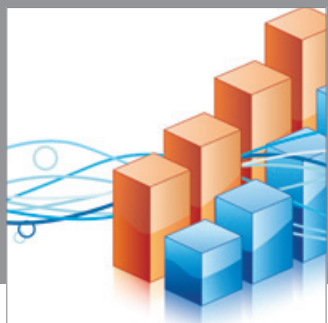

Advances in

Operations Research

mansans

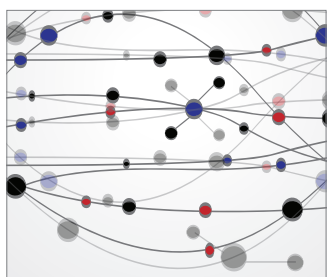

The Scientific World Journal
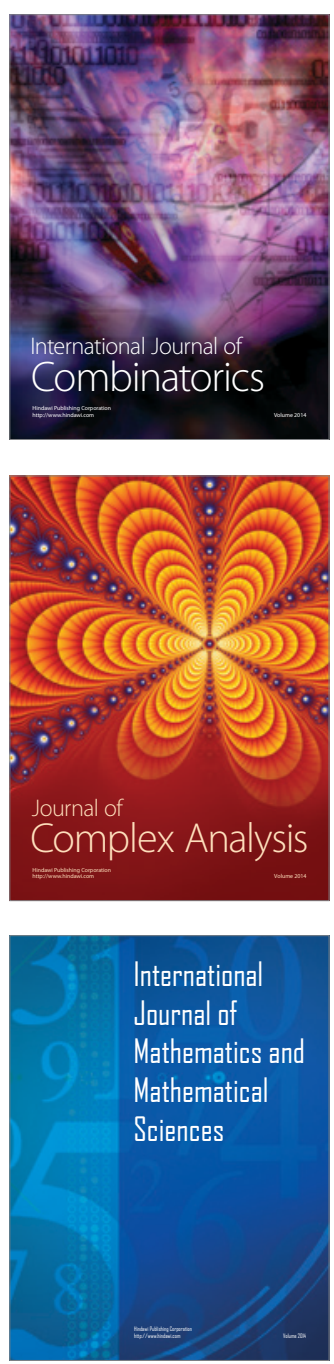
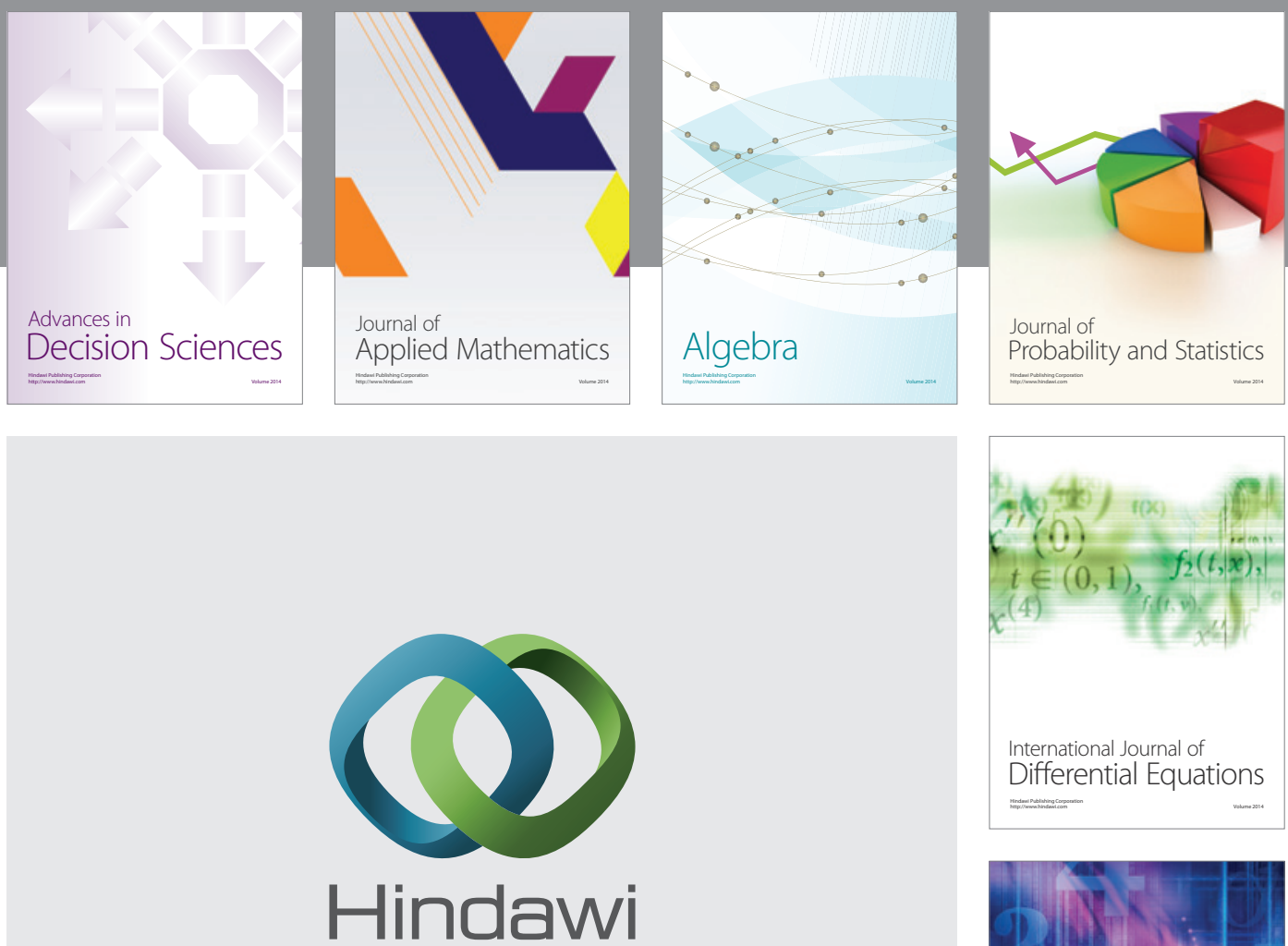

Submit your manuscripts at http://www.hindawi.com
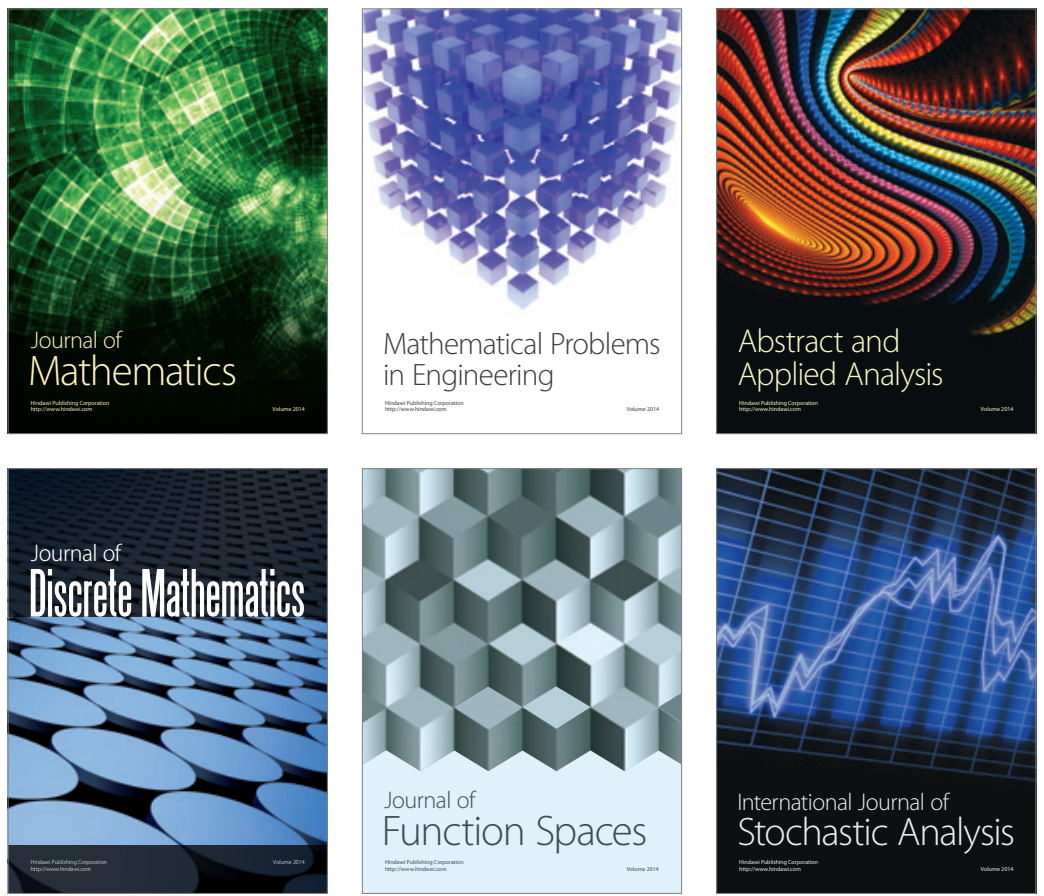

Journal of

Function Spaces

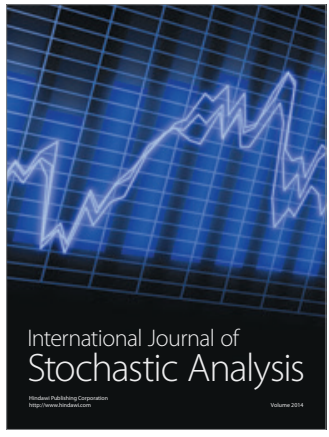

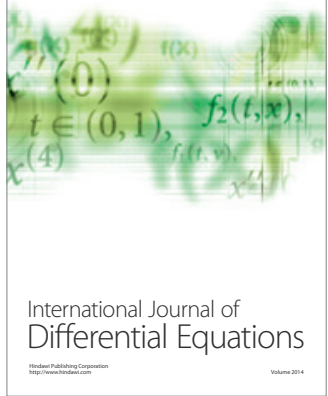
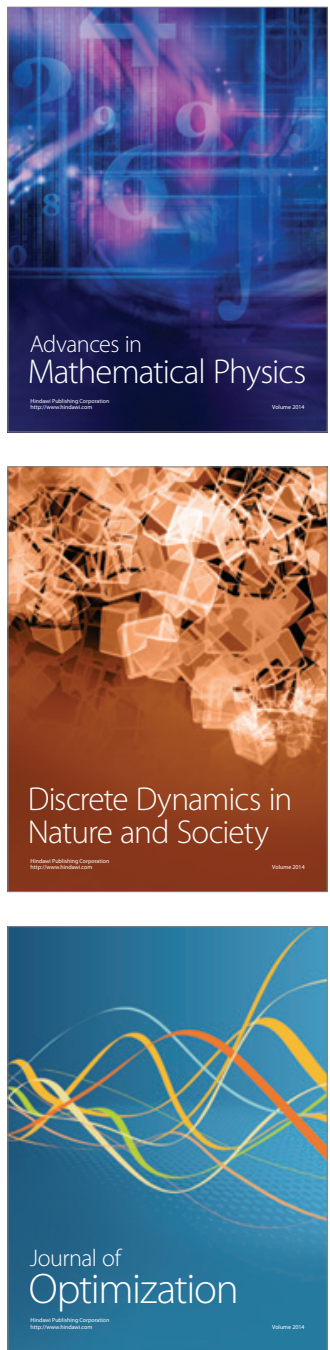